his work appears, a common reaction is likely to be "What's new?" The answer, in the case of Einstein's Mirror, is its emphasis on the way that relativity theory is rooted in experiment and, as in the authors' earlier book on the quantum theory, how it is manifested in nature.

There is a widespread misapprehension in the public mind that Einstein overthrew the existing paradigms by thought alone. There are many enthusiastic individuals who, inspired by the Einstein myth, believe that they too have tapped the secrets of the Universe by their unique insight, without need or care for experiment. Although Einstein is best known as the creator of relativity, it is less widely realized that he spent most of his life in a fruitless quest for a unified theory of gravity and electromagnetism. Hey and Walters recall Richard Feynman's comment that Einstein's great works had sprung from physical intuition and that it was "when he stopped thinking in concrete physical images and became a manipulator of equations" that Einstein's creativity ceased. Einstein's Mirror shows the physical reality of relativity and how it relates to our daily lives, often in unexpected ways.

The title of the book recalls how the young Einstein was haunted by a conundrum: would his image appear in a mirror if both he and the mirror were moving at the speed of light? Einstein solved this mirror problem with his creation of special rela-

tivity; although he was not the only scientist

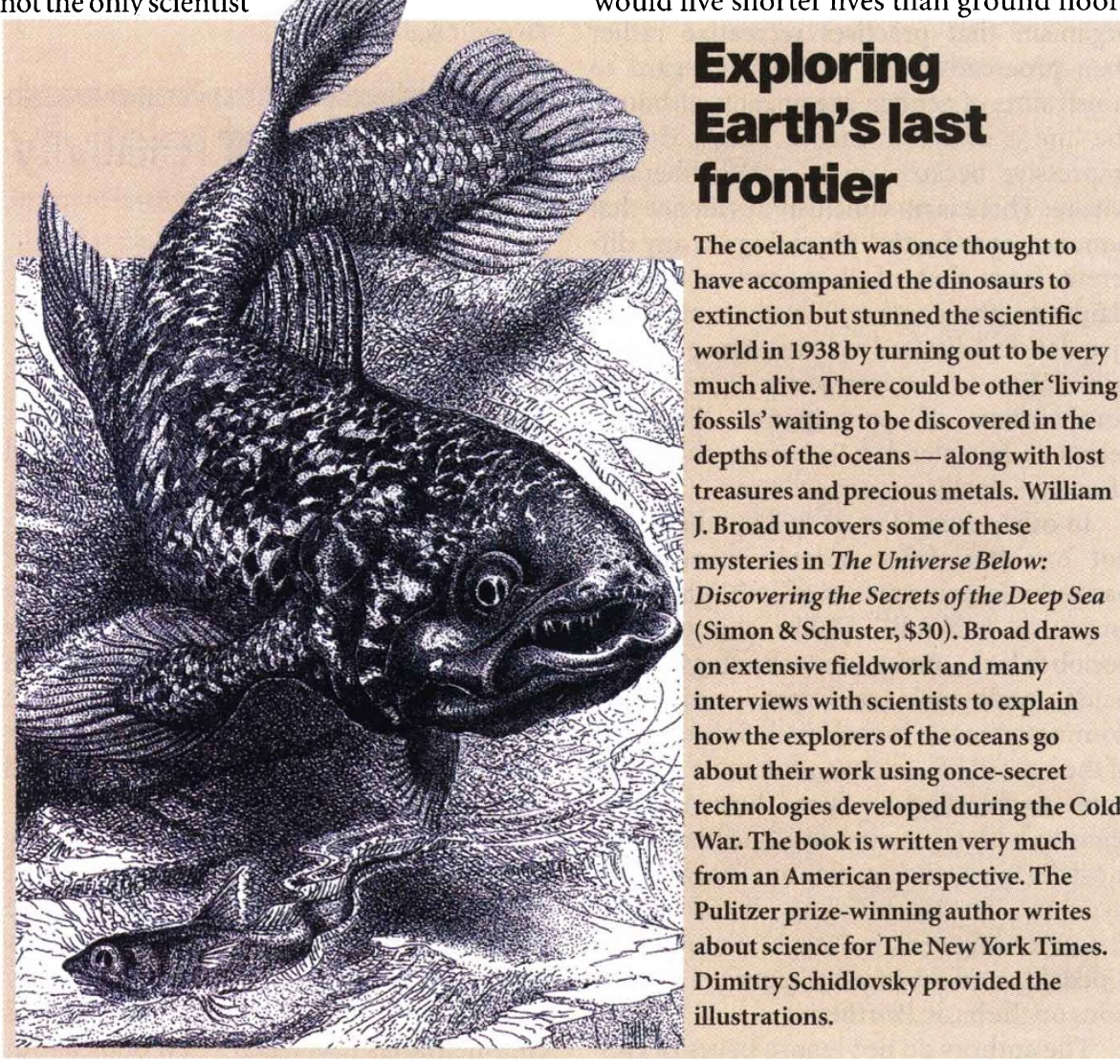

concerned with the problem of how the velocity of light might be affected by the motion of the observer, it was "Einstein alone who realized that the solution to the problem involved a radical rethinking of the concept of time". His new vision of space and time has routine applications in physics, chemistry and medicine - positron emission tomography, satellite navigation systems, particle accelerators, the famous $E=m c^{2}$, nuclear power and more are covered in the book.

The "happiest thought" of Einstein's life was that when someone is in free fall there exists, in their immediate surroundings, no gravitational field: "If the observer drops some bodies then these remain to him in a state of rest". Einstein had realized that in free fall, gravity is effectively abolished and his ideas on special relativity could be applied. Conversely, just as gravity can be 'abolished' by free fall, an artificial gravity can be created by acceleration: "Gravity and acceleration are, in a certain sense, equivalent".

This eventually led Einstein to his general theory of relativity, with its bizarre implications for the slowing of time in gravitational fields. (Incidentally, the timedilation effects of motion in high-flying aircraft and the gravitational effects of the Earth almost cancel out.) These effects have been measured on Earth with atomic clocks, but they become much greater on neutron stars, and singular in black holes. In a section called 'Life on a Neutron Star', we learn that office workers on high floors
"would live shorter lives than ground floor

\section{Exploring Earth's last} The coelacanth was once thought to have accompanied the dinosaurs to extinction but stunned the scientific world in 1938 by turning out to be very much alive. There could be other 'living fossils' waiting to be discovered in the depths of the oceans - along with lost treasures and precious metals. William J. Broad uncovers some of these mysteries in The Universe Below: Discovering the Secrets of the Deep Sea (Simon \& Schuster, \$30). Broad draws on extensive fieldwork and many interviews with scientists to explain how the explorers of the oceans go War. The book is written very much from an American perspective. The Pulitzer prize-winning author writes Dimitry Schidlovsky provided the illustrations. technologies developed during the Cold workers although they would accomplish the same amount of work in a lifetime".

In Newtonian theory, gravity acts instantaneously, whereas according to Einstein, disturbances in the gravitational field are limited by the speed of light. This implies the existence of gravitational radiation, which has not yet been detected directly, although in 1978 Russell Hulse and Joseph Taylor found indirect evidence for it in the motion of a pulsar around a companion star. According to theory, the rotating binary system should emit gravitational radiation, although it would be too weak to measure directly. However, this gravitational radiation of energy shows up as a tiny decrease in the orbital periods of the pulsar, about onetenth of a second in a year.

Fifty years ago, our vision was limited to the rainbow; then we expanded our electromagnetic senses into radio, infrared, ultraviolet, $\mathrm{X}$-ray and gamma-ray astronomies, which have led to marvels and monsters that were undreamed of. By the time of the centenary of the theory of general relativity in 2015, we are likely to be exploring the dark side of the Universe by means of gravitational-wave astronomy. If history is any guide, the resulting discoveries will be stranger than fiction. The greatest legacies of Einstein's mirror may be yet to come, but in the meantime Einstein's Mirror is an excellent introduction to the implications and applications of relativity.

Frank Close is in the Rutherford-Appleton Laboratory, Chilton, Didcot OX11 OQX, UK.

\section{New journals}

This year, Nature's annual new journals review supplement will appear in the issue of 11 September. Publishers and learned societies are invited to submit journals for review, as well as details of any eligible electronic journals, taking note of the following criteria:

- Journals that first appeared during or after June 1995 and issued at least four separate numbers by the end of May 1997 will be considered.

* Journals covering any aspect of science are eligible, although those dealing with clinical medicine and pure mathematics are excluded, as are publications of abstracts.

* Frequency of publication must be at least three times a year. The main language used must be English. Translation journals in English are, of course, eligible.

* Deadline for submission is 6 June.

When submitting journals for review, please send at least four different issues (the first, the most recent and any two others) of each title, together with full details of subscription rates. For further information please contact Peter Tallack, Nature, Macmillan Magazines, Porters South, Crinan Street, London N1 9XW, UK. Tel: +44 (0)171843 4567. e-mail: p.tallack@nature.com. 